\title{
Deep compressed sensing-based accurate blade vibration reconstruction using sub-sampled tip- timing measurements
}

\section{Zhongsheng Chen ( $\nabla$ chenzs@czu.cn )}

Changzhou Institute of Technology https://orcid.org/0000-0001-7354-0006

Hao Sheng

Hunan University of Technology

Chengwu Liu

Changzhou Institute of Technology

\section{Yeping Xiong}

University of Southampton

\section{Research Article}

Keywords: Unknown multi-band vibrations, blade tip-timing, sub-sampled vibration reconstruction, multicoset angular sampling, Deep compressed sensing

Posted Date: March 7th, 2022

DOI: https://doi.org/10.21203/rs.3.rs-1268712/v1

License: (9) This work is licensed under a Creative Commons Attribution 4.0 International License. Read Full License 


\title{
Deep compressed sensing-based accurate blade vibration reconstruction using sub-sampled tip-timing measurements
}

\author{
Zhongsheng Chen ${ }^{\mathrm{a}, \mathrm{b} *}$, Hao Sheng ${ }^{\mathrm{b}}$, Chengwu Liu ${ }^{\mathrm{a}}$, Yeping Xiong ${ }^{\mathrm{c}}$ \\ ${ }^{a}$ College of Automotive Engineering, Changzhou Institute of Technology, Changzhou 213032, China \\ ${ }^{\mathrm{b}}$ College of Electrical \& Information Engineering, Hunan University of Technology, Zhuzhou 412007, China \\ ${ }^{c}$ Faculty of Engineering and Physical Sciences, University of Southampton, Southampton SO16 7QF, UK
}

\begin{abstract}
Blade tip-timing (BTT) measurement is seriously sub-sampled due to the restricted number of BTT probes, so that true blade vibration characteristics can hardly be directly extracted. Blade vibration responses are always multi-band which can be considered to be sparse, so compressed sensing (CS) can be used to reconstruct blade vibrations. However, there is often less prior sparse information of multi-band blade vibrations, so that classical reconstruction algorithms are less effective. In order to deal with it, this paper presents an end-to-end deep compressed sensing (DCS)-based blade vibration reconstruction method which is composed of four key operations. First, angular-domain sampling is introduced to adapt complex operation conditions and a Multi-coset BTT measurement model is proposed in angular domain. Then a DCS model of BTT measurements is presented in order domain and a convolutional neural network (CNN) is specially designed. Next, a Simulink model is presented to simulate BTT sampling process under variable speeds and simulations are done to study the effects of the CNN architecture on the reconstruction accuracy. The results demonstrate that convolution layers with ReLU after BN are much beneficial for BTT vibration reconstruction and the proposed method is better than classical algorithms in terms of reconstruction accuracy and speed. In the end, experiments are carried out to validate the DCS-based reconstruction method and true vibration Engine Order can be recovered effectively without prior information. Thus the proposed method may set up a novel direction for BTT signal analysis and blade vibration monitoring.
\end{abstract}

Keywords: Unknown multi-band vibrations, blade tip-timing, sub-sampled vibration reconstruction, multi-coset angular sampling, Deep compressed sensing.

\section{Introduction}

High-speed rotating blades are widely used in modern industry, such as compressor blades, turbine blades, and fan blades. While faults or damages of rotating blades are prone to emerge due to severe operation conditions including strong vibrations, large centrifugal forces, thermal stresses, and so on. It has been reported that more than $70 \%$ of blade faults are induced by vibrations $[1,2]$. Thus on-line blade vibration monitoring is very significant for safety, reliability, and availability. Traditionally, strain gauges are mounted to measure blade vibrations [3, 4]. However, this way has several unavoidable shortcomings, including contact measurement, high cost and short lifetime. Nowadays, blade tip-timing (BTT) has been proved to be an advanced non-contact method and investigated by almost all gas turbine or aero-engine manufacturers as replacing strain gauges [5]. Chen et al. gave a comprehensive review on blade tip timing-based health monitoring [6]. Basic idea of the BTT method is to measure times of arrival (TOAs) of each blade

*Corresponding authors

E-mail address: chenzs@czu.cn(ZSC), Y.Xiong@ soton.ac.uk(YPX) 
passing each BTT sensor. When blade vibrations happen, the blades will pass BTT sensors earlier or later than ideal times. In this case, a TOA difference series will be generated for each blade and then blade vibration displacements can be calculated. In theory, BTT sampling frequency is equal to the product of the rotating frequency and the number of BTT sensors. While the number of BTT sensors is always small due to the limitations of space, cost and safety in engineering applications. Therefore, the Nyquist sampling theorem can hardly be satisfied, so that BTT vibration signals are seriously sub-sampled. In this case, true blade vibration characteristics can hardly be extracted due to frequency alias and it is very necessary to reconstruct blade vibrations.

Up to now, main challenges of BTT vibration reconstruction can be summarized as three aspects. The first one is from unstable operation conditions due to different mission profiles. In this case, the rotating speed is often not constant, which will complicate BTT vibration measurement. The second one is from multi-band vibrations. Blade vibration responses often contain multiple frequency components due to system nonlinearity, complex excitations or incipient damages. Accurate multi-band vibration reconstruction is still a difficult task. The third one is lack of prior information of multi-band vibrations. That is to say, it is hardly to obtain the number and locations of multi-frequency components due to system nonlinearity, complicated aerodynamic excitations, and so on. In particular, super-/sub-harmonic vibration responses and/or frequency modulations will appear once incipient damages happen in blades. In early studies, blade vibration responses are assumed as single band-pass signals. Under this assumption, Salhi et al. first proposed a technique to reconstruct a continuous BTT signal [7]. Chen et al. proposed to reconstruct sub-sampled blade vibrations by using the Shannon sampling theorem and wavelet packet transform [8, 9]. As for non-constant rotating speeds, Chen et al. further studied two reconstruction methods under speed fluctuations [10] and variable speeds [11], respectively. Lin et al. presented a novel reconstruction method for non-uniformly sub-sampled BTT data based on the periodically non-uniform sampling theorem [12]. Above methods are mainly based on the assumption that the target signal is band-pass and its central frequency is known in advance. Therefore, the three challenges cannot be overcome and new reconstruct methods are desirable.

For multi-band blade vibration responses, only relevant frequencies to incipient damages, rotating speed and aerodynamic excitations are dominant [13]. Thus blade vibrations can be considered to be sparse in frequency domain, which is valuable for reconstruction. As a novel sampling paradigm, compressed sensing (CS) was first proposed by Donoho et al in 2006 [14]. The CS theorem takes fully advantages of sparsity and compressibility of signals, so that we can recover certain signals from sub-sampled signals. Therefore, it is very promising to introduce the CS theorem to reconstruct sub-sampled multi-band blade vibrations. Xu and Chen et al. first proposed a CS-based method for blade vibration monitoring using BTT signals [15], and then built a CS model of BTT measurements by minimizing the $\ell_{1}$ norm [16]. Pan et al. proposed a dictionary learning method for representing sparse BTT vibration signals and then reconstructed blade vibrations by using the basis pursuit algorithm [17]. Spada et al. evaluated several conditions of CS 
for BTT signals [19]. For classical CS model, several algorithms have been proposed to obtain its unique and stable sparse solution, such as Orthogonal Matching Pursuit (OMP), Multiple Signal Classification (MUSIC), Modified Focal Underdetermined System Solver (MFOCUSS) and Basis Pursuit Denoising (BPDN). Chen et al. compared the performances of the above four reconstruction algorithms used for BTT vibration reconstruction [16]. However, most of these algorithms still depend on prior sparse information of original signals [18]. To sum up, unknown multi-band vibration reconstruction is still a current obstacle of BTT-based vibration monitoring, which has not been solved well by existing reconstruction algorithms.

In recent years, deep learning (DL) has achieved great success in many fields due to excellent learning ability, such as image processing [20], speech recognition [21]. Its most advantage is to learn the structure of a signal and obtain effective inherent information, instead of using prior knowledge. In particular, DL is being combined with CS for image reconstruction. Zisselman et al. presented a mathematical framework of compressed learning and a deep neural network (DNN) was introduced for image classification in the CS domain [22]. Ye and Han proposed a general deep learning framework called deep convolutional framelets for inverse problems of image reconstruction [23], where reconstruction performances under various conditions were greatly improved. Inspired by these studies, this paper will introduce DL into BTT vibration reconstruction and propose an end-to-end deep compressed sensing (DCS) method. To our best knowledge, it is the first to introduce DL to solve the problem of unknown multi-band vibration reconstruction using sub-sampled tip-timing measurements. The remainder of this paper is summarized as follows: In Section 2, basic principles of BTT measurement is introduced briefly and then its under-sampling character is emphasized. In Section 3, compressed sensing model of BTT vibrations is built in angular domain to adapt complex conditions. Then DCS-based BTT vibration reconstruction algorithm is described in Section 4. Numerical simulations and experiments are done to validate the proposed method in Section 5 and Section 6, respectively. In the end, some conclusions are marked in Section 7.

\section{Problem statements}

Basic principle of BTT method can be schematically shown in Figure 1. A blisk is composed of $K$ blades and $I$ BTT probes are mounted in a stationary case around the blisk. A once-per-revolution (OPR) sensor are placed in front of the rotating shaft on which one speed mark is arranged, so that timing reference signals can be generated. It is assumed that the OPR sensor is on the same radius as the first BTT probe and the OPR marker is on the same radius as the first blade. Then the angular positions of the $i^{\text {th }}(1 \leq i \leq I)$ BTT probe and the $k^{\text {th }}(1 \leq k \leq K)$ blade relative to the OPR mark are denoted as $\alpha_{i}$ and $\theta_{k}$, respectively.

The basis of BTT method is to measure times of arrival (TOAs) when each blade passes each BTT probe. When there 
are no blade vibrations under ideal conditions, TOAs of each blade are only related to rotating speed, rotating radius and its circumferential position. Once these parameters are given in advance, the TOAs can be accurately calculated which are called theoretical TOAs. While there are vibrations, the blades will pass BTT probes earlier or later than theoretical TOAs. Therefore, actual TOAs will deviate from theoretical ones, which will lead to time differences. Furthermore, these time differences are strongly related to vibration frequency and amplitude of the blade, so that they can be used to calculate blade vibration displacements. TOAs of each blade can be collected during rotation, so the BTT method can measure all-blade vibrations of the blisk.

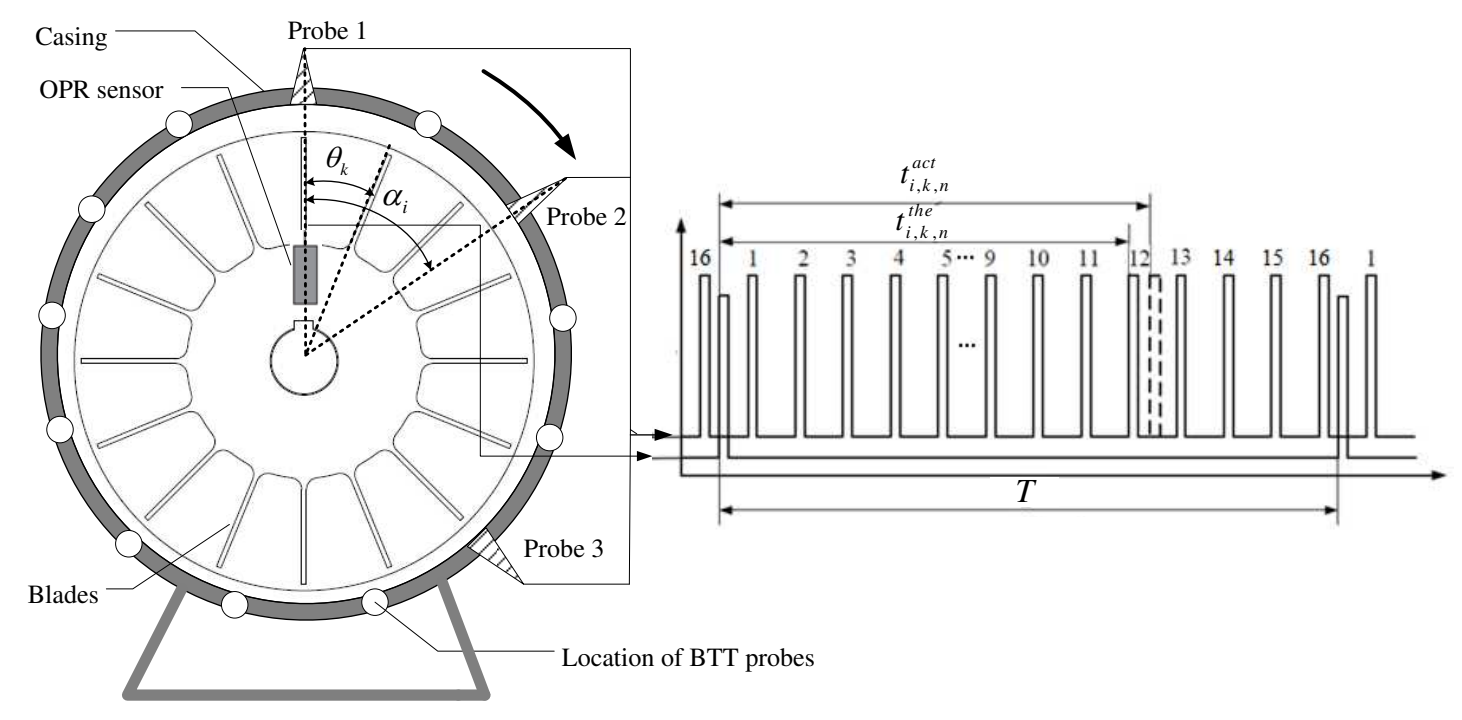

Figure 1. Basic principles of BTT method

For the sake of easy understanding, let the blisk rotate clockwise at a constant rotating speed $\left(f_{r}\right)$. When no vibrations, theoretical TOAs of the $k^{\text {th }}$ blade passing the $i^{\text {th }}$ BTT probe can be calculated as

$$
t_{i, k, n}^{\text {the }}=\left\{\begin{array}{l}
{\left[2 \pi(n-1)+\alpha_{i}-\theta_{k}\right] / 2 \pi f_{r}, \theta_{k} \leqslant \alpha_{i}} \\
{\left[2 \pi n+\alpha_{i}-\theta_{k}\right] / 2 \pi f_{r}, \theta_{k}>\alpha_{i}}
\end{array}\right.
$$

where $n$ denotes the $n^{\text {th }}$ revolution.

The corresponding actual TOAs is denoted as $t_{i, k, n}^{a c t}$, then vibration displacement of the $k^{\text {th }}$ blade measured by the $i^{\text {th }}$ BTT probe can be calculated as,

$$
d_{i, k}[n]=2 \pi f_{r} R\left(t_{i, k, n}^{a c t}-t_{i, k, n}^{\text {the }}\right)
$$

where $R$ is the rotating radius of the blade tip.

As stated before $[11,12]$, BTT vibration signals are seriously sub-sampled and 'true' vibration characteristics of a blade is difficult to directly obtain due to frequency alias. Another concern in Eqs.(1) and (2) is about the rotating speed. Under unstable operation conditions, the rotating speed cannot keep constant. In this case, theoretical TOAs are difficult to calculate and Eq.(2) can hardly be used yet to obtain vibration displacements. In addition, the sampling time interval is not a constant even though BTT probes are mounted uniformly, leading to unequally-sampled vibration signals. As 
we all know that angular-domain sampling has always been applied to deal with variable speeds [24]. It is coincident that BTT probes are mounted circumferentially, so that BTT sampling is a natural angular-domain sampling process. Thus this paper will perform BTT vibration analysis in angular domain. By this way, the effects of unstable operation conditions can be greatly reduced.

In angular domain, theoretical angles of arrival (AOAs) of the $k^{\text {th }}$ blade passing the $i^{\text {th }}$ BTT probe under no vibrations can be represented as,

$$
\theta_{i, k, n}^{\text {the }}=\left\{\begin{array}{l}
2 \pi(n-1)+\alpha_{i}-\theta_{k},(n=1,2, \cdots), \theta_{k} \leqslant \alpha_{i} \\
2 \pi(n-1)+2 \pi+\alpha_{i}-\theta_{k},(n=1,2, \cdots), \theta_{k}>\alpha_{i}
\end{array}\right.
$$

In order to illustrate the principle clearly, the rotating speed of each revolution is assumed to be constant. Then actual AOAs of the $k^{\text {th }}$ blade passing the $i^{\text {th }}$ BTT probe can be calculated as,

$$
\begin{aligned}
\theta_{i, k, n}^{a c t}= & 2 \pi(n-1)+2 \pi f_{n}\left(t_{i, k, n}^{a c t}-\sum_{p=1}^{n-1} T_{p}\right) \\
= & 2 \pi(n-1)+\omega_{n} \Delta t_{n}^{i k}
\end{aligned}
$$

where $T_{p}, f_{n}$ are the rotating period of the $p^{\text {th }}$ revolution and frequency of the $n^{\text {th }}$ revolution, respectively, $\omega_{n}=2 \pi f_{n}$ and $\Delta t_{n}^{i k}$ is the measured passing time of the $k^{\text {th }}$ blade passing the $i^{\text {th }}$ BTT probe in the $n^{\text {th }}$ revolution.

Combining Eq.(3) with Eq.(4), angular-domain sampled vibration displacements of the $k^{\text {th }}$ blade measured by the $i^{\text {th }}$ BTT probe can be calculated as,

$$
y_{i, k}[n]=\left(\theta_{i, k, n}^{a c t}-\theta_{i, k, n}^{\text {the }}\right) R
$$

Based on Eqs.(3) (5), we can see that angular-domain vibration displacements are strongly related to the rotating angular speed in the $n^{\text {th }}$ revolution. In engineering applications, $\omega_{n}$ in Eq.(4) is often defined as $\omega_{n}=2 \pi /\left(t_{n}-t_{n-1}\right)$ to reduce angular bias when the rotating speed changes slowly, where $t_{n}, t_{n-1}$ are the ending times of the $n^{\text {th }}$ and $(n-1)^{\text {th }}$ revolutions, respectively. However, $\omega_{n}$ cannot be looked as a constant again if the rotating angular speed changes rapidly. In this case, calibration methods need to be explored, which are out of the scope of this paper. In particular, it should be pointed out that Eqs.(3) (5) still hold for constant rotating speeds.

\section{Compressed sensing model of BTT vibrations in angular domain}

True vibration displacement of the $k^{\text {th }}$ blade in angular domain is assumed to be $y(\theta)$. According to the sampling process in Figure 1, we can see that $y(\theta)$ is sampled for $I$ times during each revolution. Furthermore, the above sampling procedure can be looked as $I$-channel parallel sampling with fixed angular delay. Then such a sampling scheme can be equivalent to a multi-coset angular sampling (MCAS) process [25], as shown in Figure 2. It is assumed 
that $L$ virtual BTT probes are uniformly mounted and the number of each probe is set from 1 to $L$ clockwise. Here $L$ satisfies $L \geq 2 E_{o}^{\max }$ in order to perfectly reconstruct vibrations of the blade, where $E_{o}^{\max }$ is the maximum vibration Engine Order (EO) of the blade.

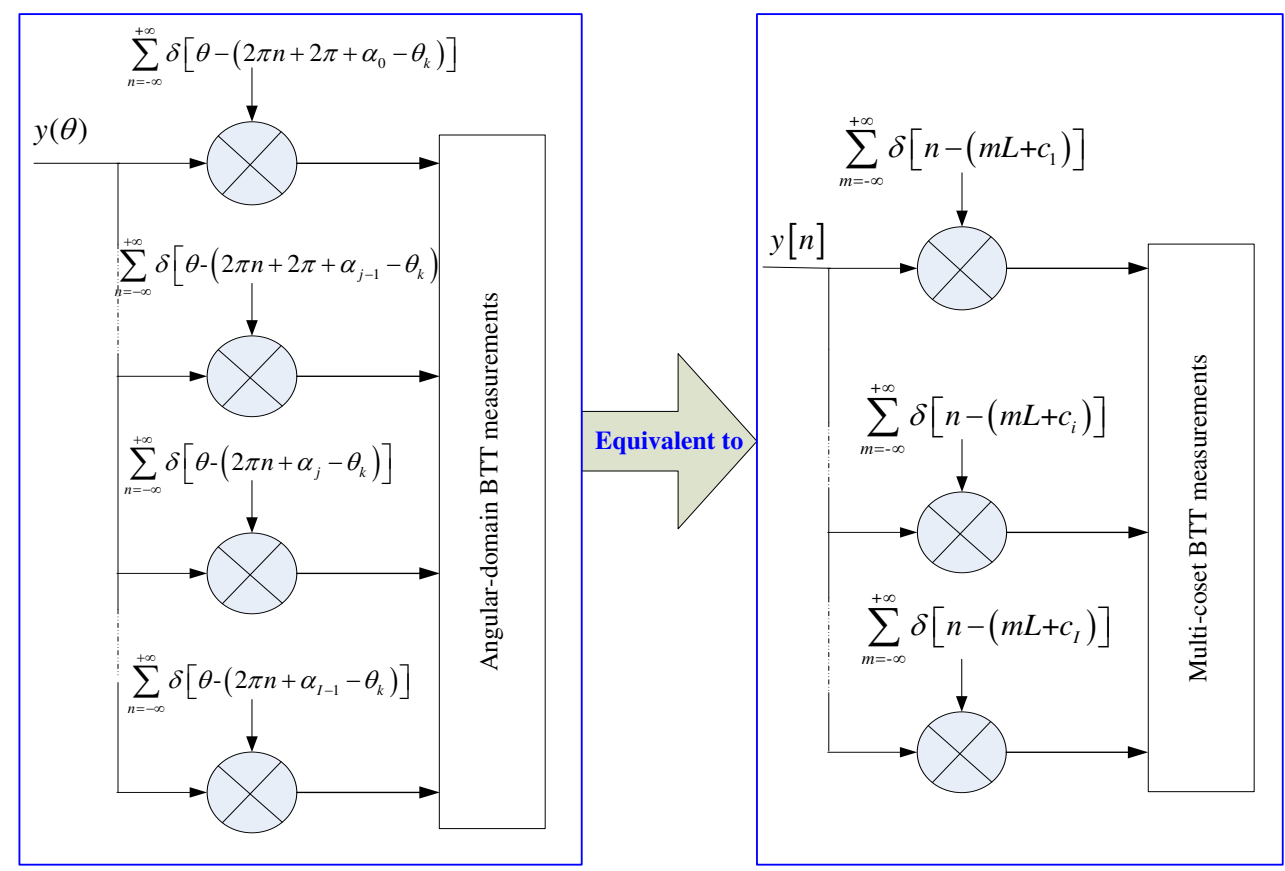

Figure 2. MCAS scheme of angular-domain BTT vibration signals

Then the Nyquist-sampled vibration signals in angular domain can be represented as,

$$
y[n]=y(n \Delta \theta), \Delta \theta=2 \pi / L
$$

At the same time, the $I$ actual BTT probes are considered to choose from the above $L$ virtual BTT probes, which are called as a MCAS pattern $(L, I, C)$. Here $C=\left\{c_{i}: 1 \leq i \leq I\right\}$ is the set of $I$ BTT probes and $c_{i}$ is the number of the $i^{\text {th }}$ probe $\left(0 \leq c_{1}<c_{2} \cdots<c_{I} \leq L-1\right)$. According to the MCAS scheme, angular-domain sampled vibrations of the $i^{\text {th }}$ BTT probe can be represented as,

$$
\bar{y}_{i}[n]=y\left[n L+c_{i}\right], i=1, \ldots, I
$$

where the sampling angle interval of each BTT probe is denoted as $\Delta \theta_{I}=L \Delta \theta$.

In practice, $I$ is always much less than $L$ due to the limitations of space and cost, so $\bar{y}_{i}[n]$ is also sub-sampled in angular domain. It should be pointed out that $y[n]$ can be used to perfectly recover true EOs of the blade. Thus the remaining problem is how to correctly reconstruct $y[n]$ based on $\bar{y}_{i}[n]$.

For multi-band blade vibrations, they are also sparse in order domain. Thus it is probable to reconstruct $y[n]$ by using sub-sampled signals $\left(\bar{y}_{i}[n]\right)$. Firstly, the relation between $y[n]$ and $\bar{y}_{i}[n]$ in order domain can be built as follows [17]. 


$$
\bar{Y}_{i}(O)=\frac{e^{j 2 \pi O c_{i}}}{L} \sum_{k=1}^{L} e^{-j \frac{2 \pi}{L}\left(k-\frac{L+1}{2}\right) c_{i}} Y\left(O-\left(k-\frac{L+1}{2}\right)\right)
$$

where $O$ denotes the order, $O \in[-1 / 2,1 / 2]$ and $\bar{Y}_{i}(O), Y(O)$ are the discrete Fourier transforms of $\bar{y}_{i}[n], y[n]$, respectively.

Physical meaning of Eq. (8) can be described as that the entire order band $(Y(O))$ is equally divided into $L$ sub-bands and the bandwidth is equal to 1, as shown in Figure 3. The $l^{\text {th }}$ sub-band can be represented as follows.

$$
Y_{l}(O)=Y\left(O-\left(l-\frac{L+1}{2}\right)\right), l=1,2, \cdots L
$$

Then, $\bar{Y}_{i}(O)$ can be looked as the weighed sum of sub-bands of $Y(O)$. Furthermore, for $I$ BTT probes, Eq. (8) can be expressed as the following matrix form.

$$
\overline{\mathbf{Y}}(O)=\boldsymbol{\Phi} \mathbf{Y}(O)
$$

where, $\overline{\mathbf{Y}}(O)=\left[\bar{Y}_{1}(O) \exp \left(-j 2 \pi O c_{1}\right), \cdots \bar{Y}_{I}(O) \exp \left(-j 2 \pi O c_{I}\right)\right]^{T}, \quad \mathbf{Y}(O)=\left[Y_{1}(O), \cdots Y_{L}(O)\right]^{T}$,

$$
\boldsymbol{\Phi}=\frac{1}{L}\left[\begin{array}{ccccc}
\exp \left(-j 2 \pi c_{1}(1-(L+1) / 2) / L\right) & \cdots & \exp \left(-j 2 \pi c_{1}(l-(L+1) / 2) / L\right) & \cdots & \exp \left(-j 2 \pi c_{1}(L-(L+1) / 2) / L\right) \\
\vdots & \vdots & \vdots & & \vdots \\
\exp \left(-j 2 \pi c_{i}(1-(L+1) / 2) / L\right) & \cdots & \exp \left(-j 2 \pi c_{i}(l-(L+1) / 2) / L\right) & \cdots & \exp \left(-j 2 \pi c_{i}(L-(L+1) / 2) / L\right) \\
\vdots & \vdots & \vdots & \vdots & \vdots \\
\exp \left(-j 2 \pi c_{I}(1-(L+1) / 2) / L\right) & \cdots & \exp \left(-j 2 \pi c_{I}(l-(L+1) / 2) / L\right) & \cdots & \exp \left(-j 2 \pi c_{I}(L-(L+1) / 2) / L\right)
\end{array}\right] .
$$

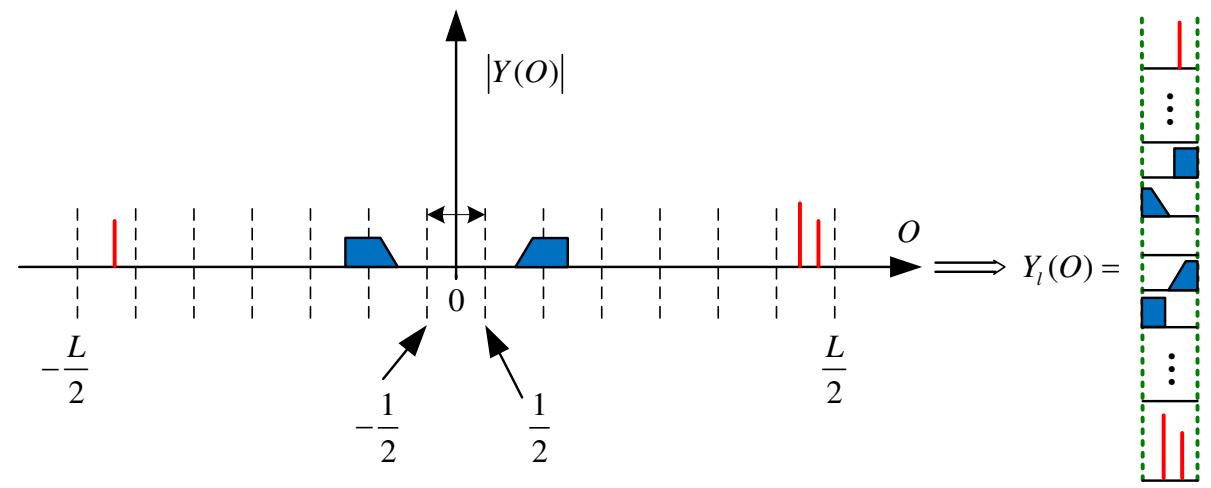

Figure 3. The sub-band division of $Y(O)$

In Eq.(10), $\overline{\mathbf{Y}}(O)$ can be calculated by $I$ actual BTT measurements in angular domain. Thus the reconstruction task is transformed to find the unknown $\mathbf{Y}(O)$ based on the known $\overline{\mathbf{Y}}(O)$ and $\boldsymbol{\Phi}$. Moreover, $\mathbf{Y}(O)$ is sparse, so it only has few non-zero rows. In this case, the above MCAS of BTT vibration signals becomes a CS pattern, which can be represented as,

$$
\begin{array}{ll}
\mathbf{P 0}: & \min \|\mathbf{Y}(O)\|_{0} \\
& \text { s.t. } \overline{\mathbf{Y}}(O)=\mathbf{\Phi Y}(O)
\end{array}
$$

where $\|\cdot\|_{0}$ is the $\ell_{0}$-norm. 


\section{Deep compressed sensing-based BTT vibration reconstruction}

\subsection{Basic framework}

As for the classical CS problem as Eq.(11), the key is the restriction of Restricted Isometry Property (RIP) which is defined for the measurement matrix [26]. In order to solve Eq.(11), several algorithms have been presented, such as Orthogonal Matching Pursuit (OMP), Multiple Signal Classification (MUSIC), Basis Pursuit Denoising (BPDN), and Modified Focal Underdetermined System Solver (MFOCUSS). However, these existing reconstruction algorithms are often limited by its strong assumption on sparsity and slow optimization process. In order to accurately recover unknown multi-band vibrations using sub-sampled BTT measurements, this paper proposes an end-to-end DCS framework which is composed of five components: measurement of TOAs, calculation of AOAs, multi-coset measurement model, CS model and DNN, as shown in Figure 4. There are four key operations: angular sampling, multi-coset sampling, compressed sensing and learning sensing. Key advantages of this framework include: i) Complex conditions can be handled by transferring time domain to angular domain. ii) Unknown multi-band vibrations can be reconstructed by introducing multi-coset sampling. iii) High-fidelity and fast reconstruction can be achieved by using deep learning algorithms.

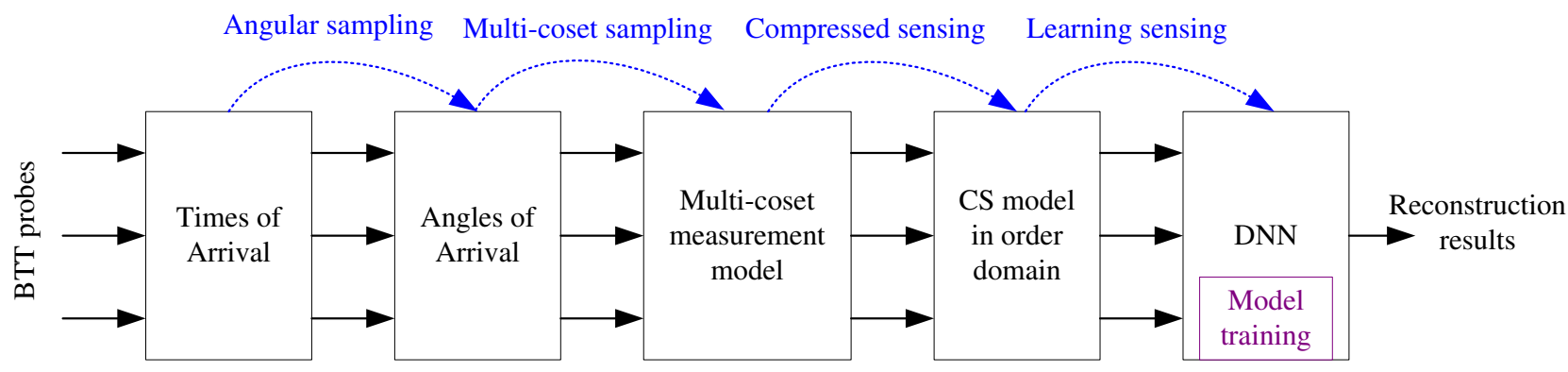

Figure 4. Schematic diagram of the proposed DCS framework for BTT signals

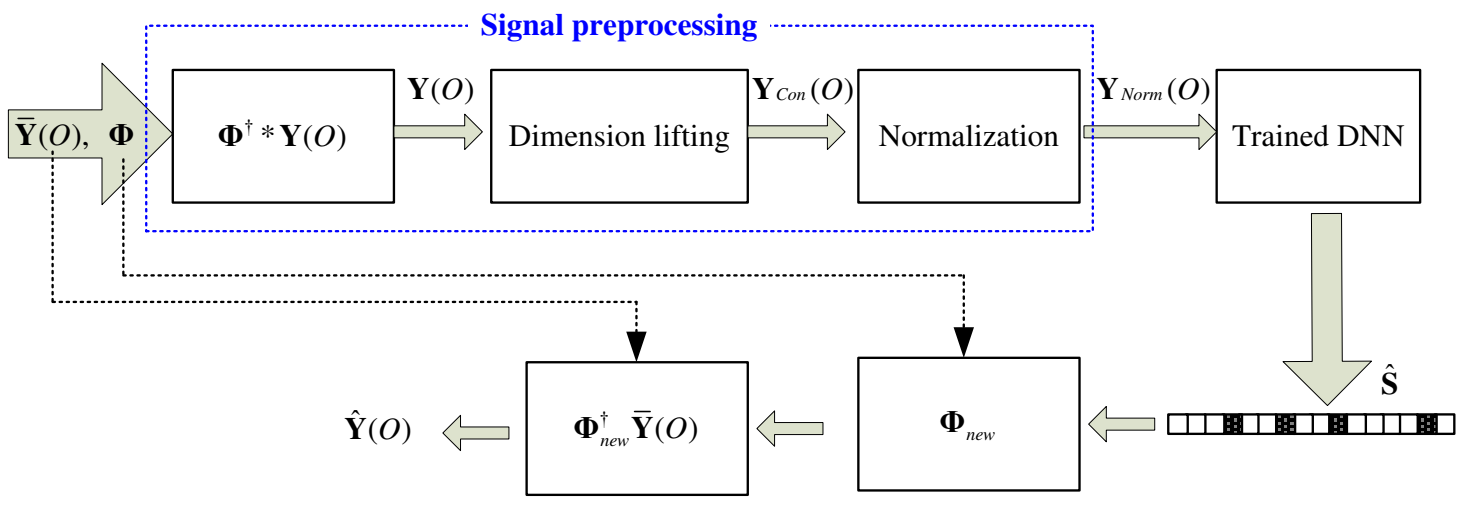

Figure 5. Schematic diagram of the proposed DCS-based reconstruction model

Next, the key problem is how to solve Eq.(11) by learning methods. In order to deal with it, this paper first proposes a DCS model by introducing DNN, as shown in Figure 5. The initial inputs of the proposed DCS model are $\overline{\mathbf{Y}}(O)$ and $\boldsymbol{\Phi}$, 
both of which are obtained from the $I$ BTT probes. Firstly, we should carry out signal preprocessing of $\overline{\mathbf{Y}}(O)$. The pseudo-reconstruction signal of $\mathbf{Y}(O)$ is calculated as follows.

$$
\mathbf{Y}(O)=\left(\boldsymbol{\Phi}^{H} \boldsymbol{\Phi}\right)^{-1} \boldsymbol{\Phi}^{H} \overline{\mathbf{Y}}(O)
$$

where $\boldsymbol{\Phi}^{H}$ is the transpose of $\boldsymbol{\Phi}$.

Obviously, $\mathbf{Y}(O)$ is a complex signal, so it cannot be directly input to the DNN model. In order to deal with it, we will concatenate the real and imaginary parts of $\mathbf{Y}(O)$ to form a higher-dimension matrix $\tilde{\mathbf{Y}}_{c o n}(O)$.

$$
\tilde{\mathbf{Y}}_{\text {con }}(O)=[\operatorname{Re}(\mathbf{Y}(O)), \operatorname{Im}(\mathbf{Y}(O))]
$$

Furthermore, a normalization operation is carried out on $\tilde{\mathbf{Y}}_{c o n}(O)$ in order to accelerate convergence of the training process. By this way, each element of $\tilde{\mathbf{Y}}_{c o n}(O)$ is normalized between $[0,1]$.

$$
\mathbf{Y}_{\text {Norm }}(O)=\operatorname{Norm}\left(\mathbf{Y}_{C o n}(O)\right)
$$

Next a trained DNN is utilized to generate an $L$-dimension band occupancy status vector $\hat{\mathbf{S}}$ whose elements $\left(\hat{S}_{i}\right)$ are equal to 0 or 1 . That is to say,

$$
\hat{S}_{i}=\left\{\begin{array}{l}
0, \text { if vacant band } \\
1, \text { if non-vacant band }
\end{array}\right.
$$

Here, $\hat{\mathbf{S}}$ shows the sparsity of $\mathbf{Y}(O)$. Then the new sensing matrix $\boldsymbol{\Phi}_{\text {new }}$ is formed by retaining the columns of $\boldsymbol{\Phi}$ with indices corresponding to $\hat{S}_{i}=1$ and the reconstruction of $\mathbf{Y}(O)$ can be calculated as follows.

$$
\hat{\mathbf{Y}}(O)=\boldsymbol{\Phi}_{\text {new }}^{\dagger} \overline{\mathbf{Y}}(O)
$$

where $\boldsymbol{\Phi}_{\text {new }}^{\dagger}$ is the pseudo-inverse of $\boldsymbol{\Phi}_{\text {new }}$.

In the end, the target $Y(O)$ can be obtained by re-arranging $\hat{\mathbf{Y}}(O)$, leading to the order spectra of $y[n]$. In this case, true blade vibration characteristics can be recovered. It can be seen that the core of the proposed DCS model is to design and train a proper DNN model.

\subsection{Design of a specific CNN model}

Model architecture: As we all know that convolutional neural network (CNN) is an efficient deep learning model whose structure is similar to biological neural networks. CNN has many advantages such as simple structure, less training parameters and adaptability and it has been widely used in many fields. Thus this paper aims to design a specific CNN model and its basic architecture is shown in Figure 6, which is composed of one input layer, four convolution layers, one pooling layer, two fully connected layers and one output layer. In this CNN model, the rectified 
linear unit (ReLU) activation function and the Max pooling are used. Traditionally, a Batch Normalization layer is always placed before the activate function to reduce internal covariate shift. By this way, DNN training can be dramatically accelerated [27]. Recently, some studies pointed out that the BN could be placed before the ReLU activation function since non-negative responses of ReLU made the weighted layer updated in a suboptimal way [28]. In addition, Hinton et al. first proposed to use 'Dropout' to reduce overfitting on a small training set by preventing complex co-adaptations [29]. Dropout was always applied to the fully connected layers as a regularization trick. Later, Park and Kwak analyzed the effect of Dropout in the convolution layers and proved the powerful generalization performance [30]. In order to deal with the problems of small dataset and overfitting, this paper proposes to define a convolution layer by introducing a BN layer and a Dropout layer, which is shown in Figure 7 . In the specific convolution layer, the BN and Dropout are combined together and then placed after the ReLU activation function. At the same time, a small dropout probability is applied for the Dropout layer.

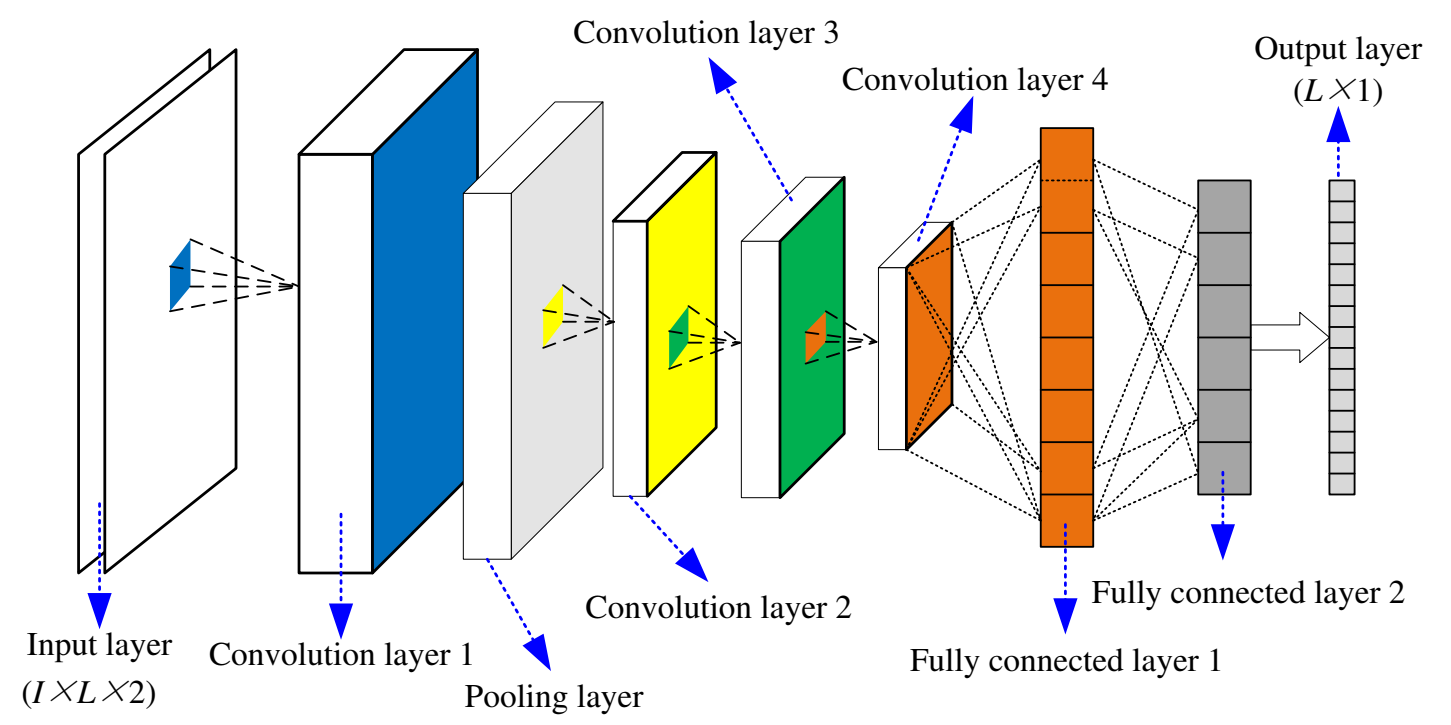

Figure 6. Basic architecture of the specific $\mathrm{CNN}$ model

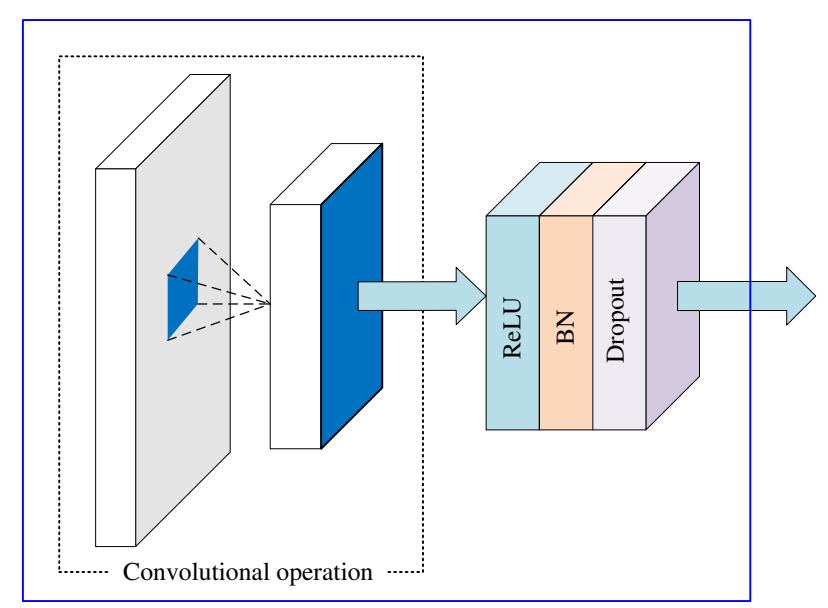

Figure 7. Schematic architecture of the specific convolution layer

Architecture parameters: After many trials, final architecture parameters of the extended CNN model are listed in 
Table 1 , including the filter sizes, the number of filters in each layer. The input layer is a $I \times L \times 2$ matrix corresponding to $\tilde{\mathbf{Y}}_{c o n}(O)$. The kernel sizes of the first to the fourth convolution layers are $1 \times 150,1 \times 50,1 \times 20$ and $1 \times 10$, respectively. The kernel size of the Max pooling layer is $2 \times 2$. The numbers of neurons in the first and second fully connected layers are 1024 and 128, respectively. The output is a $L \times 1$ vector corresponding to the band occupancy status vector $(\hat{\mathbf{S}})$. These parameters can be iteratively updated by training the specific CNN model with given data sets.

Table 1. Architecture parameters of the specific CNN model

\begin{tabular}{cccc}
\hline Layer & Filter size & Number of filters & Output dimension \\
\hline Input layer & $/$ & $/$ & $I \times L \times 2$ \\
Convolution layer 1 & $1 \times 100$ & 128 & $I \times L \times 128$ \\
Max pooling layer & $2 \times 2$ & $/$ & $/$ \\
Convolution layer 2 & $1 \times 50$ & 64 & $I \times L \times 64$ \\
Convolution layer 3 & $1 \times 20$ & 32 & $I \times L \times 32$ \\
Convolution layer 4 & $1 \times 10$ & 16 & $I \times L \times 16$ \\
Fully connected layer 1 & $/$ & $/$ & $1024 \times 1$ \\
Fully connected layer 2 & $/$ & $/$ & $128 \times 1$ \\
Output layer & $/$ & & $L \times 1$ \\
\hline
\end{tabular}

Model training: Training a CNN model is a process of optimizing kernels in convolution layers and weights in fully connected layers by minimizing differences between output predictions and given labels on a training dataset. Training process of the $\mathrm{CNN}$ model consists of two stages: A forward propagation where input data are transformed into output through different layers, and a backward propagation where both kernels and weights are updated according to the loss value through backward propagation with an optimization algorithm.

Obviously, two key elements of training the CNN model are the loss function and the optimizer. A loss function is applied to measure the compatibility between output predictions and given labels. In this paper, the output of band occupancy status is an $L$-D vector whose elements are equal to 0 or 1 , so the forward network can be looked as a multi-label classifier. Commonly used loss function for multiclass classification is cross entropy. As for this task of multi-label binary classification, the training loss function is defined as the binary cross entropy which is calculated as,

$$
L(\mathbf{S}, \mathbf{S})=-\sum_{i=1}^{L}\left[S_{i} \log S_{i}+\left(1-S_{i}\right) \log \left(1-S_{i}\right)\right]
$$

where $S_{i} \in \mathbf{S}$ and $\hat{S}_{i} \in \hat{\mathbf{S}}$ are the actual and predicted occupancy status of the $i^{\text {th }}$ band.

Then an optimizer can be used to iteratively update the kernels and weights of the network so as to minimize the loss function. The gradient of the loss function provides the direction of the steepest increase rate and each learnable parameter is updated in the negative direction of the gradient with a learning rate. In practice, the learning rate $(\eta)$ is one of the most important hyperparameters to be set before the training starts. After being trained, the CNN model is 
used to infer the band occupancy status of unknown multi-band BTT vibration signals in real-time. Once $\hat{\mathbf{S}}$ is obtained, $Y(O)$ can be reconstructed based on Eq.(16).

\section{Numerical simulations}

\subsection{Simulink-based BTT signal simulation}

In order to validate the proposed DCS method, numerical simulations are done to generate BTT training and testing datasets. Here a rotational blade is assumed to be a cantilever beam and only the first-order bending mode is considered. Under the simplified assumption, dynamic behavior of the blade can be represented by a single-degree-of-freedom (SDOF) lumped-parameter model and its vibration equation is written as follows [31].

$$
m_{e q} \ddot{y}(t)+c_{e q} \dot{y}(t)+k_{e q} y(t)=F(t)
$$

where $m_{e q}, c_{e q}, k_{e q}$ are the equivalent mass, damp and stiffness, respectively. $y(t)$ denotes blade vibration displacement. $F(t)$ denotes the vibration excitation.

In order to simulate variable conditions, the rotating frequency of the blade is denotes as $f_{r}(t)$, varying linearly with time. Here $f_{r}(t)$ is represented as

$$
f_{r}(t)=f_{0}+\frac{f_{e}-f_{0}}{T_{s}} t
$$

where $f_{0}, f_{e}$ are the rotating frequencies at the starting time and the ending time, respectively. $T_{s}$ is the total simulation time. Furthermore, three synchronous excitations are assumed to act on the system. That is to say, the vibration excitation can be written as,

$$
F(t)=\sum_{i=1}^{3} A_{i} \sin \left(2 \pi E O_{i} f_{r}(t) t+\varphi_{i}(t)\right)
$$

where $E O_{i}(i=1, \ldots, 3)$ denoted the $i^{\text {th }}$ Engine Order and $A_{i}, \varphi_{i}(t)$ are the corresponding amplitudes and phases.

Next the BTT sampling process in angular domain is simulated in Matlab/Simulink environment and the whole model is shown in Figure 8(a), which is composed of two parts, namely the system model and angular-domain sampling process. Firstly, the SDOF model in Eq. (18) is built as Figure 8(b). Then the angular-domain sampling process of thirteen BTT probes is built by using the 'Hit Crossing' Block and the 'Switch' Block in the Simulink. The details of the 'Sensor set 1' is shown in Figure 8(c), which is the same as those of 'Sensor set 2' and 'Sensor set 3'. The key is to determine each angular sampling time. For the rotational blade, its angular position is denoted as $\theta(t)=\theta_{0}+2 \pi f_{r}(t) t$. When the blade tip is detected by the $i$ th BTT probe, the angular sampling time in the $n$th revolution should satisfy the following condition, 


$$
2 \pi f_{r}(t) t=\alpha_{i}-\theta_{0}+2 \pi n
$$

Furthermore, each angular sampling time can be calculated by $\sin \left(2 \pi f_{r}(t) t\right)=\sin \left(\alpha_{i}-\theta_{0}\right)$, where the left part is carried out by using the 'Chirp Signal' Block and the right part is set as the threshold of the 'Hit Crossing' Block.

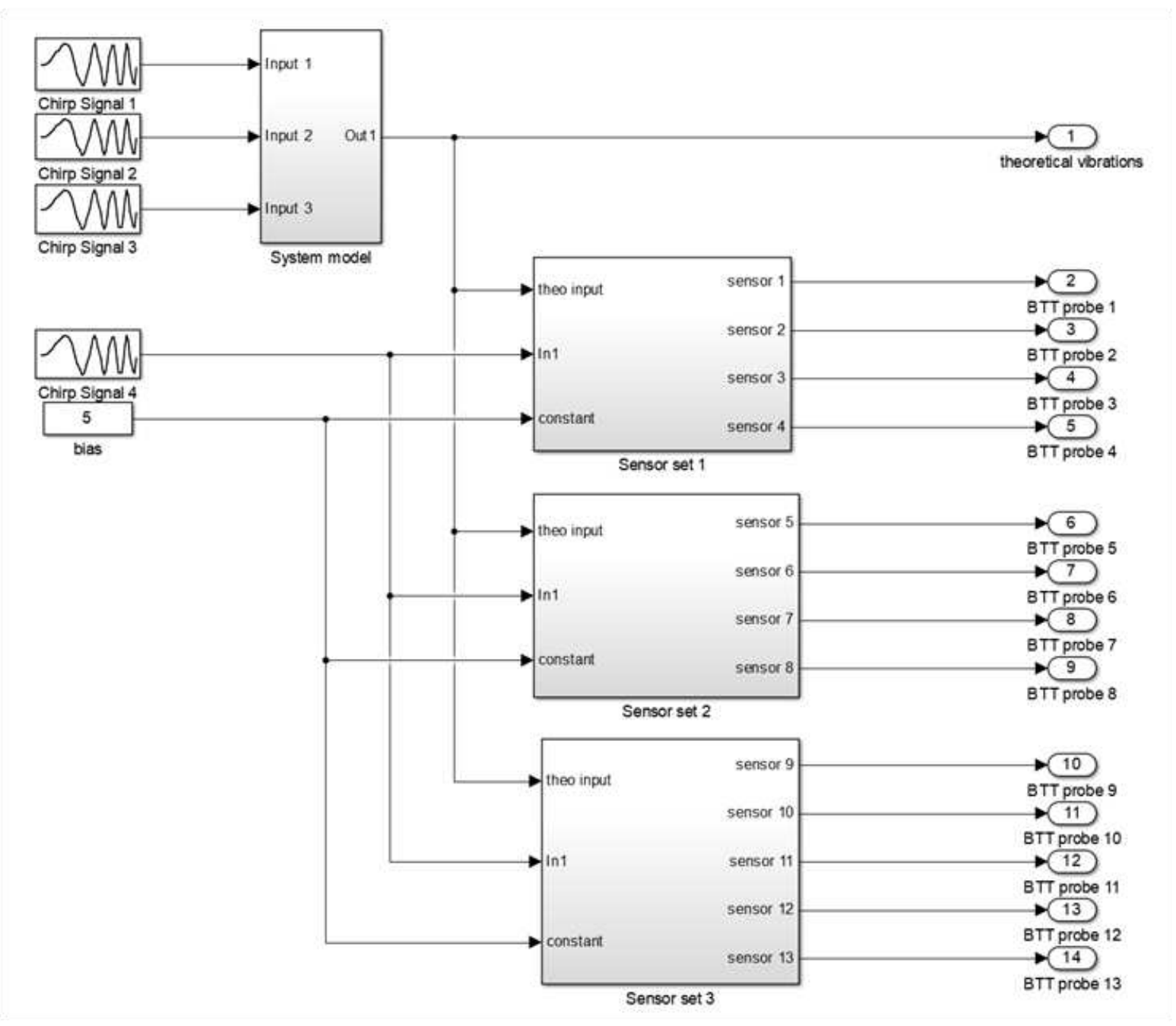

(a) The whole simulation model

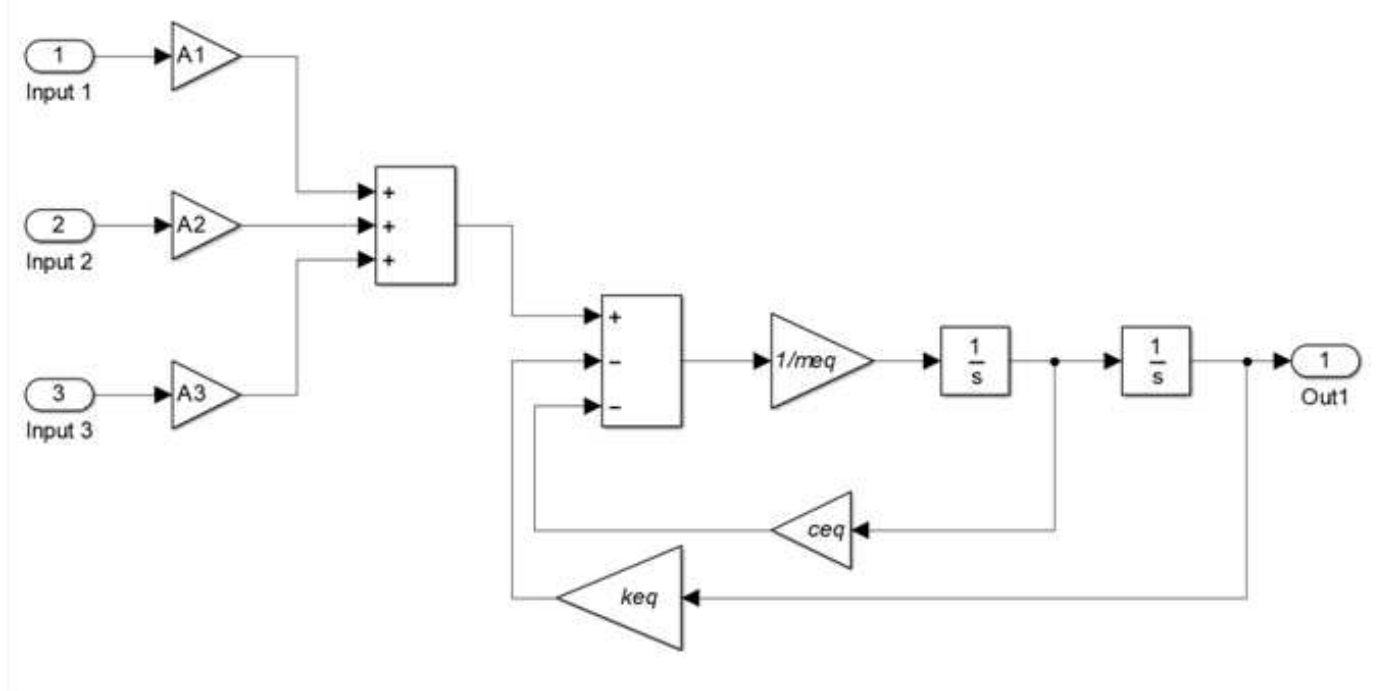

(b) Details of the 'System model' module 


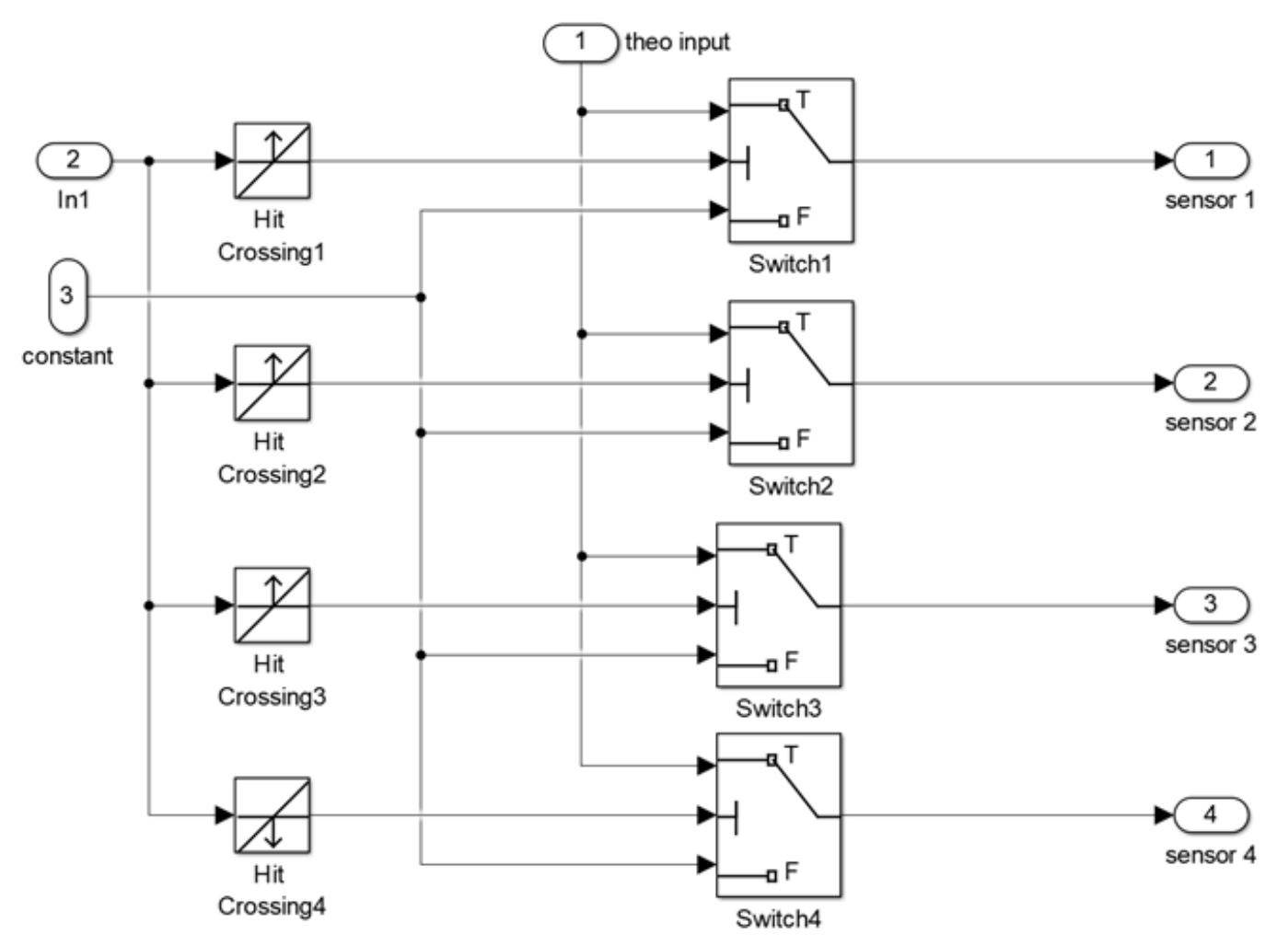

(c) Details of the 'Sensor set 1' module

Figure 8. Simulink model of BTT sampling process in angular domain

Table 2. Selected parameters in the Simulink model

\begin{tabular}{|c|c|}
\hline Parameters & Values \\
\hline$\left\{m_{e q}, c_{e q}, k_{e q}\right\}$ & $\left\{0.1 \mathrm{~kg}, 10 \mathrm{~N} \cdot \mathrm{s} / \mathrm{m}, 4 \times 10^{5} \mathrm{~N} / \mathrm{m}\right\}$ \\
\hline$\left\{f_{0}, f_{e}\right\}$ & $\{50 \mathrm{~Hz}, 200 \mathrm{~Hz}\}$ \\
\hline$T_{s}$ & $50 \mathrm{~s}$ \\
\hline$\left\{A_{1}, A_{2}, A_{3}\right\}$ & $\{4,3,2\}$ \\
\hline$\left\{E O_{1}, E O_{2}, E O_{3}\right\}$ & $\{1,2,5\}$ \\
\hline$\left\{\varphi_{1}(t), \varphi_{2}(t), \varphi_{3}(t)\right\}$ & $\{0,0,0\}$ \\
\hline$L$ & 13 \\
\hline$\left\{\alpha_{1}-\theta_{0}, \alpha_{2}-\theta_{0}, \ldots, \alpha_{13}-\theta_{0}\right\}$ & $\{\pi / 13,3 \pi / 13, \ldots, 25 \pi / 13\}$ \\
\hline Learning rate $(\eta)$ & 0.001 \\
\hline Optimizer & Adam \\
\hline
\end{tabular}

Key simulation parameters are listed in Table 2. Based on these parameters and the Simulink model in Figure 8, angularly-sampled blade vibration displacements by each BTT probe can be obtained under linear variation of rotating 
speeds. Firstly, angular-domain vibration signal sampled by the thirteen BTT probes is shown in Figure 9(a) and its order spectrum is plotted in Figure 9(b). The Nyquist sampling theorem is satisfied when $L=13$, so the three synchronous excitations can be captured accurately.
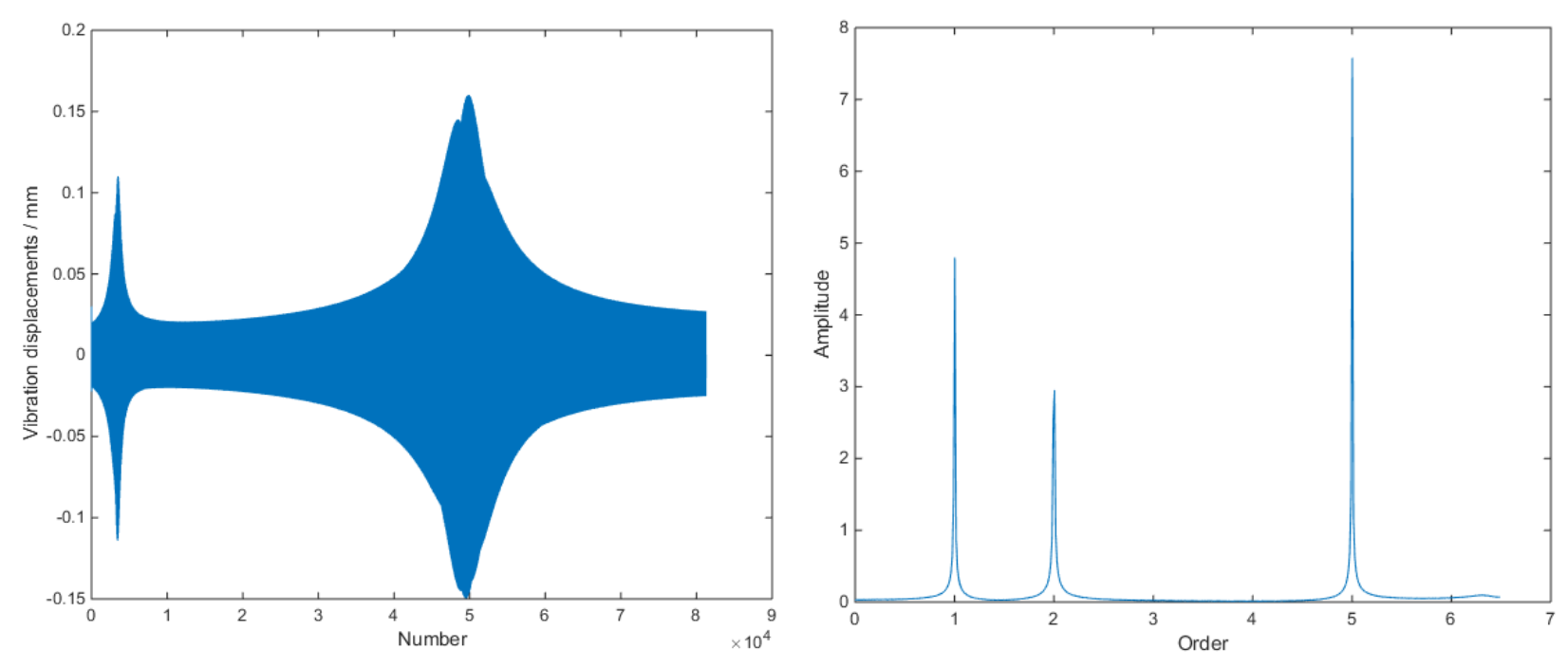

Figure 9. Sampled vibration displacements by thirteen BTT probes: (a) angular-domain signal and (b) its order spectrum

Next it needs to generate training and testing samples to evaluate the DCS model. The number of actual BTT probes is chosen as $I=4$ and $C$ is randomly generated for each sample. For each MCAS pattern, $(L, I, C)$, angular-domain samples $\left(\overline{\mathbf{y}}=\left(\bar{y}_{1}[n], \ldots, \bar{y}_{I}[n]\right)\right)$ are obtained by adding stochastic Gaussian noises with different signal-to-noise ratios (SNRs) and the corresponding band occupancy status vector $(\hat{\mathbf{S}})$ is labeled. Then each pair of $(\overline{\mathbf{y}}, \hat{\mathbf{S}})$ constitutes a sample. The total numbers of training and testing samples are equal to 7000, respectively. The Adam algorithm is used as the optimizer and the learning rate is equal to 0.001 . In order to evaluate the reconstruction performance, a metric of reconstruction accuracy is defined as follows.

$$
R_{a}=\frac{\text { number of correct reconstructions }}{\text { number of test samples }}
$$

\subsection{Effects of the specific CNN architecture}

As we all know that the architecture of a $\mathrm{CNN}$ has strong relations to its performance. Therefore, we investigate the effects of the CNN architecture on the reconstruction accuracy. Firstly, the size of filter in each convolutional layer is considered. Four different sizes of filters are studied under different SNRs, i.e. 10-5-5-5, 50-25-25-25, 100-50-20-10 and 150-50-20-10, and the corresponding reconstruction accuracies are compared in Figure 10(a). It can seen that: i) For each case, the reconstruction accuracy increases with the SNR, ii) Filters with a larger width perform better as compared to those with a smaller width, iii) Among the four sizes of filters, the size of $\{100-50-20-10\}$ has the best reconstruction 
accuracy which will be selected as the baseline CNN architecture as shown in Table 1. Next, in order to testify the improved convolutional layer, the convolutional layer with ReLU after BN is used and the results are compared in Figure 10(b). We can see that using improved convolutional layer achieves better reconstruction performance. In addition, other architecture parameters may also affect the reconstruction accuracy and no more tautology will be provided here due to the limitation of length.

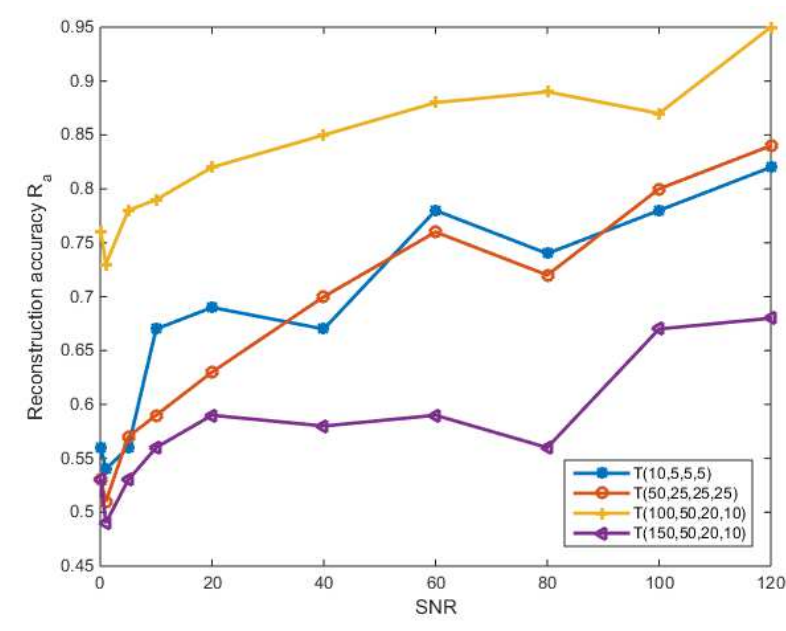

(a) different filter sizes

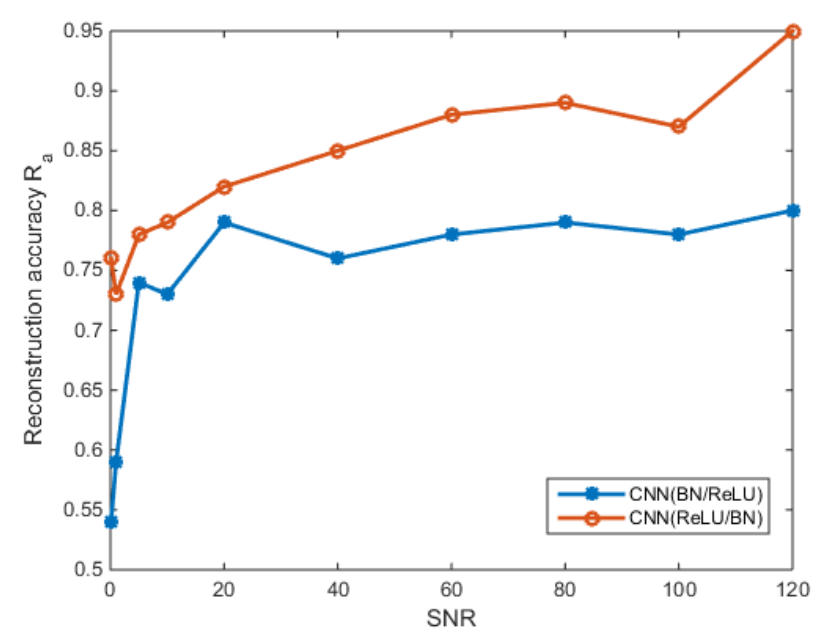

(b) different convolution layers

Figure 10. Reconstruction accuracies under different $\mathrm{CNN}$ architectures

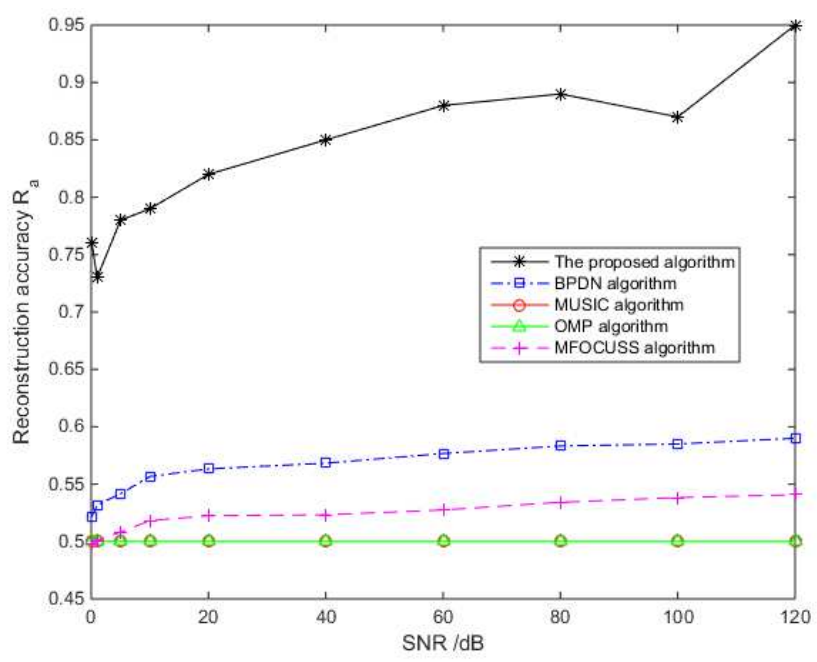

Figure 11. Comparison of the proposed method with classical CS reconstruction algorithms

\subsection{Comparisons with classical reconstruction algorithms}

As mentioned before, OMP, MUSIC, BPDN and MFOCUSS algorithms have been used to solve the CS problem. In the previous work [17], these four algorithms have been studied for compressed sensing of blade tip-timing vibration signals under variable speeds. In this section, we will compare the proposed method with these four algorithms and the simulation results are shown in Figure 11 under different SNRs. We can see that the reconstruction accuracy of the proposed algorithm is much superior to those of the four classical reconstruction algorithms. In addition, it should be emphasized that the iteration times of training the CNN model is just equal to 5, so the proposed method also has the 
best reconstruction speed. Moreover, more training samples may further improve the reconstruction accuracy of the proposed method.

\section{Experimental validations}

\subsection{Experimental set-up and datasets}

To further validate the proposed DCS reconstruction method, an experimental set-up of BTT-based vibration measurement of rotating blades is built as Figure 12. The whole experimental system is composed of a supporting base, an electrical motor, a testing bladed disk, a magnetic exciter, four BTT probes, a once-per-revolution reference sensor and a protection cover with uniform diversion grid. The electrical motor was mounted under the supporting base. BTT probes were fixed around the bladed disk through the holes in the protection cover and the angle between two adjacent BTT probes is equal to $6^{\circ}$. The OPR sensor is mounted near the center of the bladed disk.

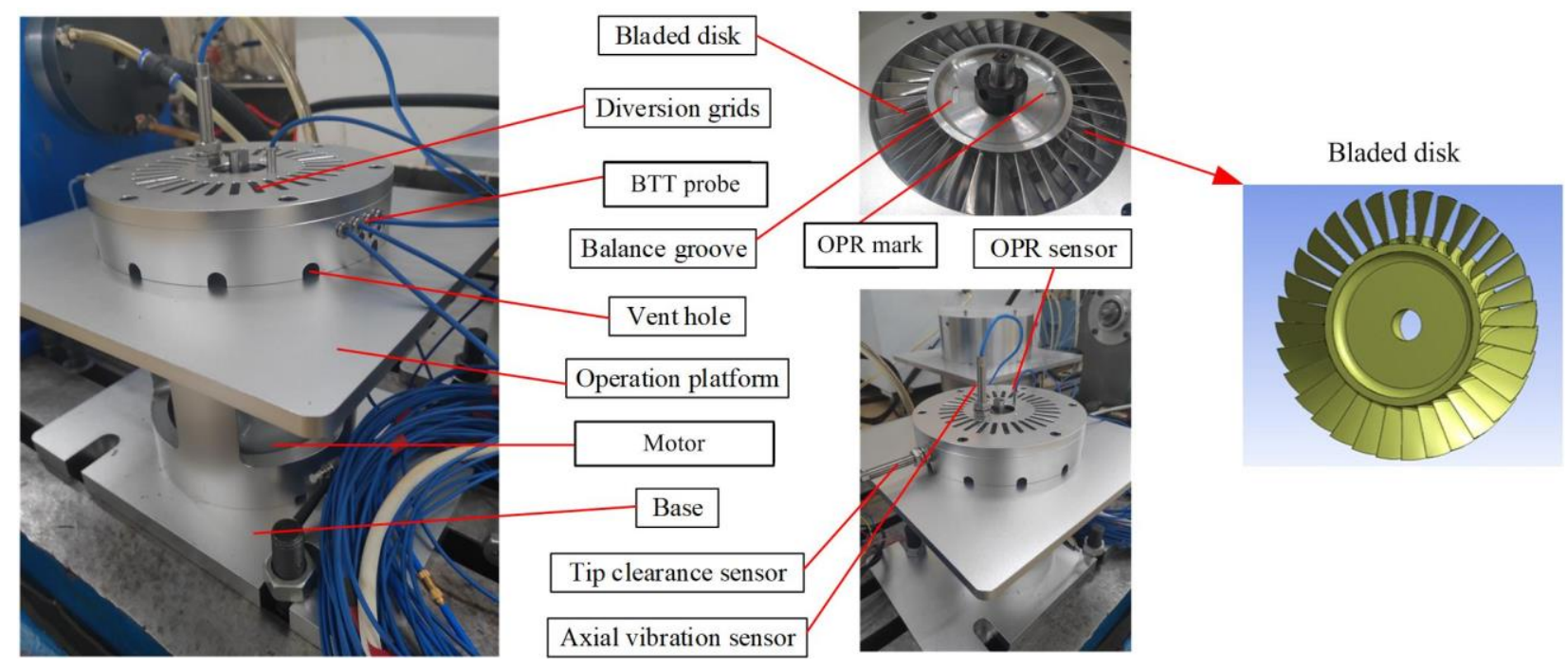

Figure 12. Experimental set-up

Table 3. Key experimental parameters

\begin{tabular}{cc}
\hline Parameters & Values \\
\hline Blade material & $40 \mathrm{Cr}$ steel \\
Blade length & $34 \mathrm{~mm}$ \\
Blade width & $11 \mathrm{~mm}$ \\
Blade tip thickness & $3 \mathrm{~mm}$ \\
Blade tip radius & $69 \mathrm{~mm}$ \\
Number of blades & 32 \\
Number of BTT probes & 4 \\
Number of OPR sensors & 1 \\
Angle of adjacent BTT probes & $6^{\circ}$ \\
Variable rotating speed & $5000 \sim 11400 \mathrm{rpm}$ \\
Acceleration time & $200 \mathrm{~s}$ \\
\hline
\end{tabular}


In order to simulate actual blades in aero-engines, the diversion grid is used as static blades and twisted blades with complex tip profiles are applied. There were 32 twisted blades on the bladed disk, which were labeled from 1 to 32. Vibration excitations are generated by permanent magnets mounted near the blades and different configurations of permanent magnets can result in different excitations. In the end, a BTT measurement system is utilized to collect BTT signals for analysis and validation. In summary, key experimental parameters are listed in Table 3.

At the beginning of the experiment, the rotating speed increases from start to 5000rpm. Then the range of variable rotating speed is set as $5000 \mathrm{rpm} \sim 11400 \mathrm{rpm}$ and the total acceleration time is equal to $200 \mathrm{~s}$. Thus the rotating speed doesn't change rapidly. During the whole acceleration process, OPR signals and TOAs are collected by the BTT measurement system, which are used to calculate angular-domain blade vibration displacements. The four BTT probes are numbered 1\#, 2\#, 3\# and 4\#. The first blade passing the 1\# BTT probe is numbered 'Blade 1' and then other blades are numbered from 'Blade 2' to 'Blade 32' along the direction of rotation. According to installation angles of the four BTT probe, $L$ in the MCAS scheme as Figure 2 is equal to $360 / 6=60$ and $I=4, C=\{0,1,2,3\}$. Then the MCAS pattern is represented as $(60,4,\{0,1,2,3\})$. Here Blade 4 is random selected as the target blade.

BTT vibration displacements of Blade 4 measured by $1 \#$, 2\#, 3\# and 4\# probe are shown in Figure 13 . It can be seen that vibration displacement increases significantly during 8000 9000 revolutions. At the same time, the corresponding speed range is equal to $8000 \sim 8500 \mathrm{rpm}$. Furthermore, the Campbell diagram of each blade is shown in Figure 14 and we can see that vibration EOs during $8000 \sim 8500 \mathrm{rpm}$ is equal to 15 . Therefore, it is obvious to find that synchronous vibration occurs during 8000 8500 revolutions and measured vibration signal during 8000 9000 revolutions is shown in Figure 15. Multiple groups of vibration signals in angular domain are collected to prepare training and testing datasets, which will be used to testify the proposed DCS method. It must be pointed out that the reconstructed order band is equal to $[-30,30]$. In this case, although there seems only to be an EO (e.g. 15), the sparsity in order domain is equal to 2 (-15 and 15). Therefore, experimental datasets are made from double-band vibration signals.

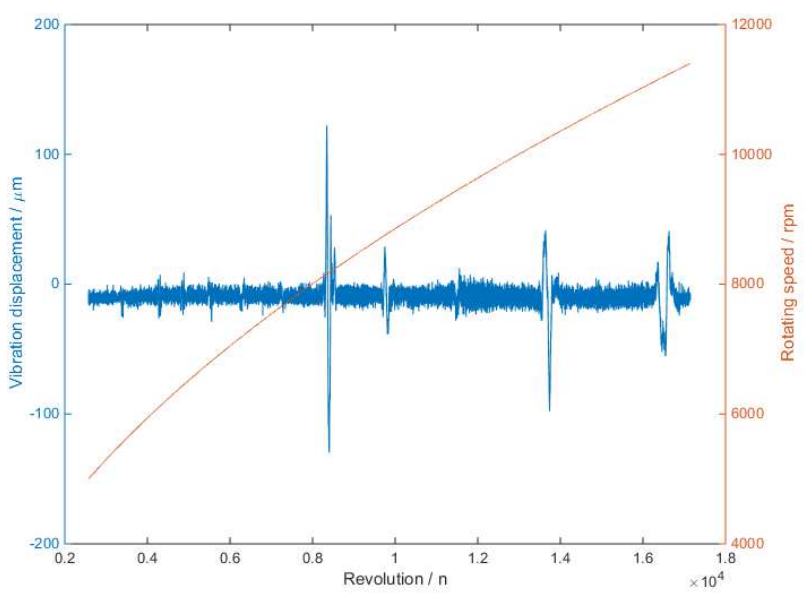

(a) Vibration signal of $1 \#$ probe

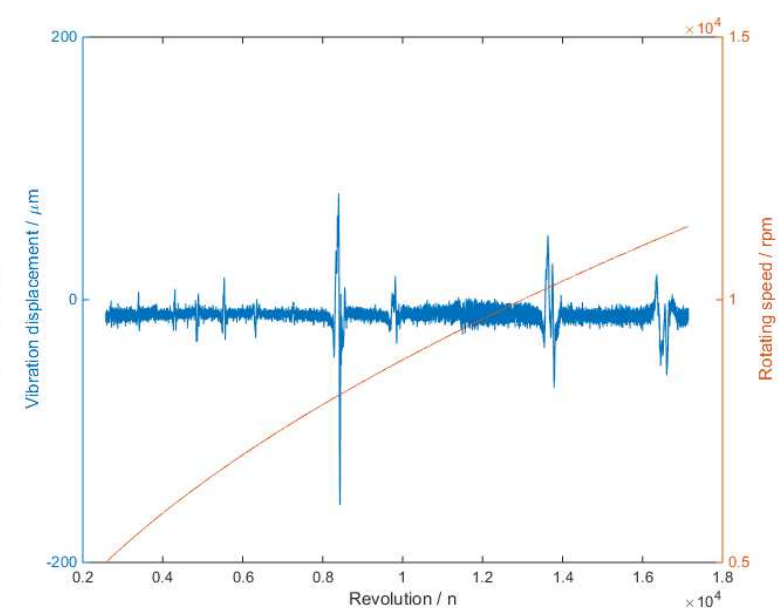

(b) Vibration signal of 2\# probe 


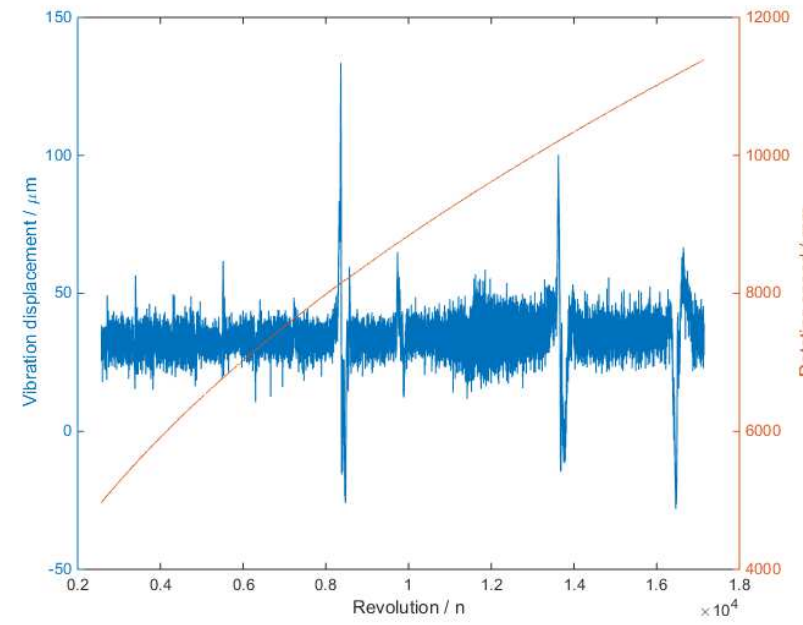

(c) Vibration signal of 3\# probe

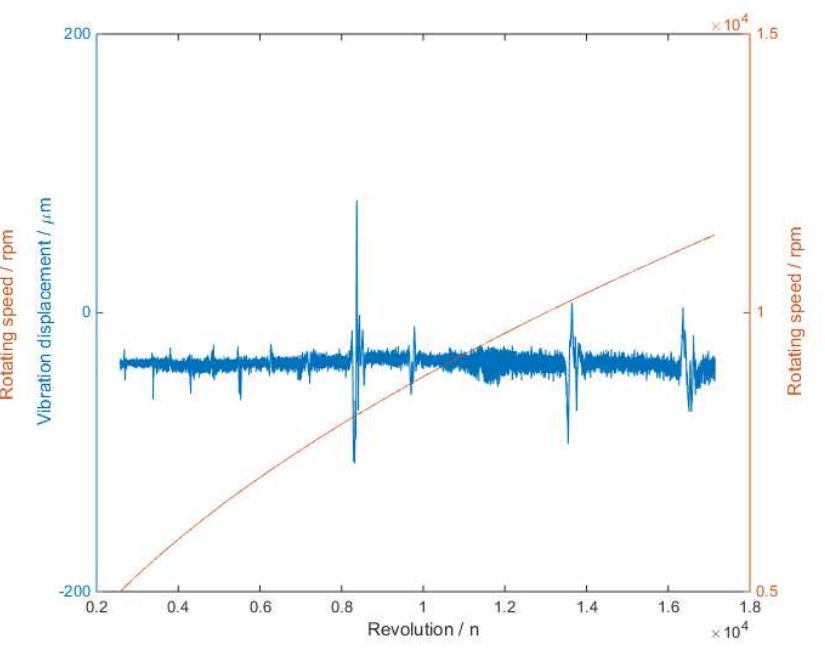

(d) Vibration signal of 4\# probe

Figure 13. BTT vibration displacements of Blade 4 by four probes

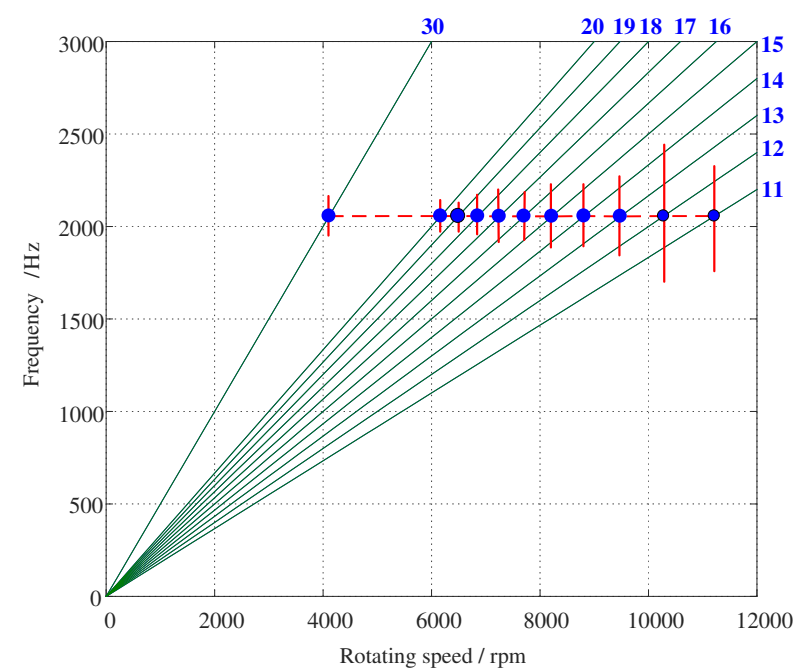

Figure 14. The Campbell diagram of Blade 4

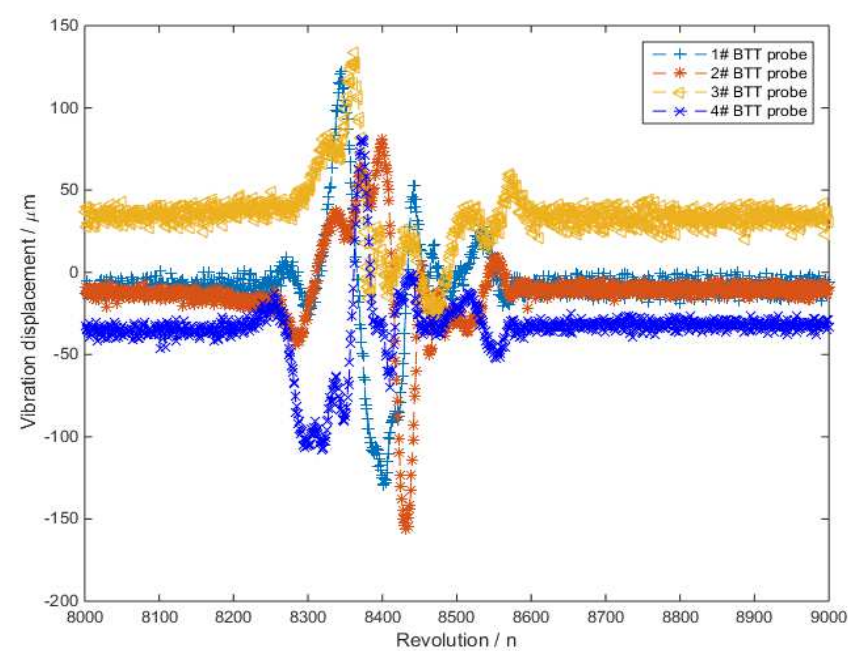

Figure 15. Vibration signals during 8000 9000 revolutions

\subsection{Validation of the proposed DCS method}

Firstly, $\overline{\mathbf{Y}}(O)$ is calculated by using measured vibration signals of four actual BTT probes and $\boldsymbol{\Phi}$ is obtained based on the arrangement of four BTT probes, as defined in Eq.(10). Then the preprocessing process including Eq.(12) Eq.(14) is carried out to obtain the input of the specific CNN model. According to the MCAS scheme, the entire order band (e.g. $[-30,30])$ of blade vibration is equally divided into 60 sub-bands and the dimension of the band occupancy status vector $(\hat{\mathbf{S}})$ is equal to 60 . Based on them, training and testing datasets can be constructed. For each dataset, the number of both training and testing samples is equal to 4000. Then training samples are used to train the specific CNN model and the iteration time is set as 5. Here model architecture parameters listed in Table 1 are utilized according to aforementioned simulation results. Finally, testing samples are used to calculate the reconstruction accuracy $R_{a}$. 
The relation between the reconstruction accuracy $\left(R_{a}\right)$ and the learning rate $(\eta)$ is shown in Figure 16(a). It can be seen that $R_{a}$ will reach the maximum value when $\eta \approx 0.005$. Then the trained DCS model with $\eta=0.005$ is used to recover the order spectra of Blade 4 in testing samples and typical reconstruction result is shown in Figure 17. It should be emphasized that the reconstruction process doesn't use any prior information of order bands.

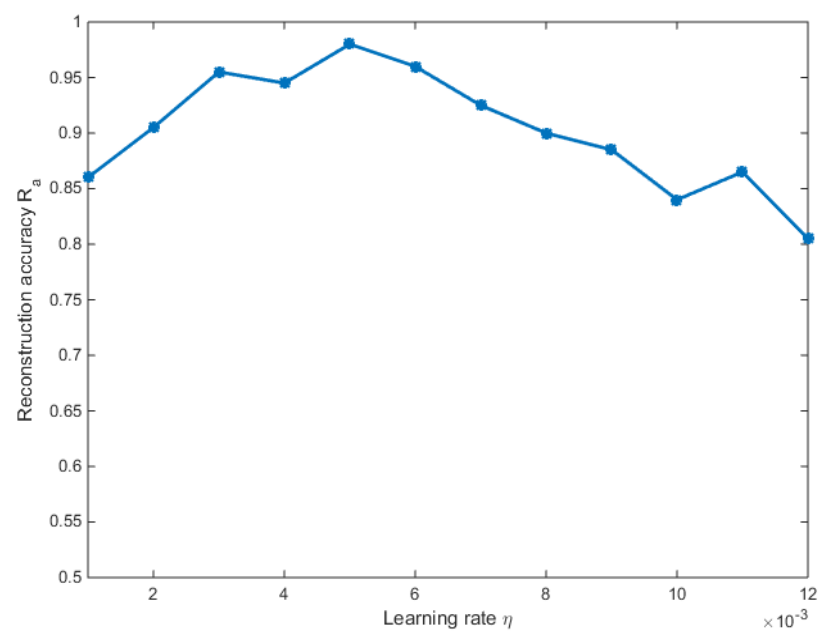

Figure 16. Effects of the learning rate

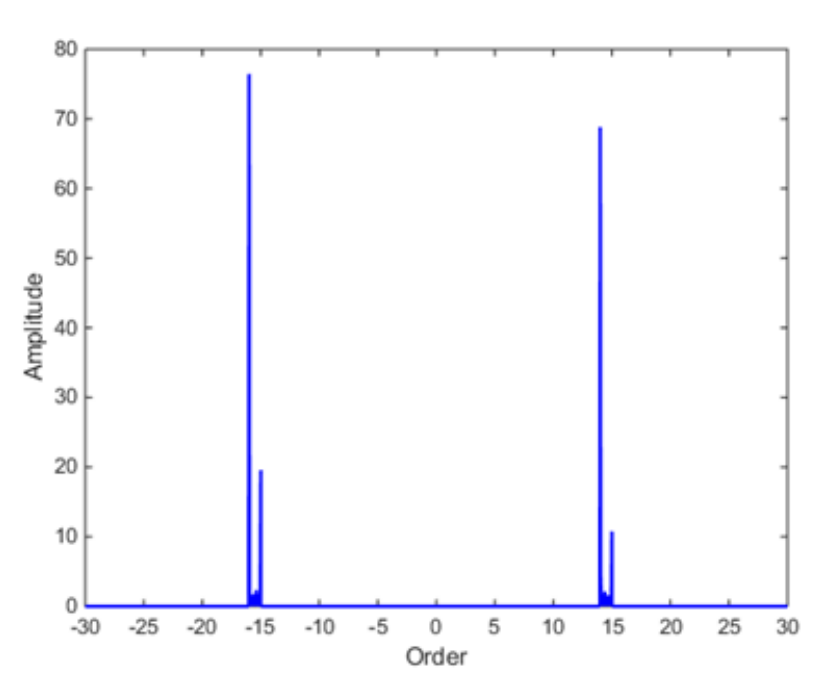

Figure 17. Reconstruction results of vibration EOs

It can be seen from Figure 16 and Figure 17 that: 1) The reconstruction accuracy is close to 98\%; 2) True vibration EOs can be reconstructed effectively by using sub-sampled BTT measurement. Meanwhile, there are small errors which may be due to manufacturing and measurement errors; 3) The iteration time of training process is just equal to 5 and the calculation time of testing process is very short, so the reconstruction speed is generally faster than that of classical CS reconstruction algorithms. In particular, if prior information of the sparsity in this experiment is imagined to be unknown, classical CS reconstruction algorithms are difficult to be used here. Therefore, comparison between the proposed DCS method classical CS reconstruction algorithms is not done here. At the same time, similar comparison has been achieved as Figure 11 .

\section{Conclusions}

Blade vibrations are often sparse in frequency or order domain, so CS can be used to reconstruct multi-band blade vibrations. However, there is no prior information of the number and locations of multi-band vibrations, so that classical sparse reconstruction algorithms are less effective. To deal with this challenge, this paper presents a novel DCS model for BTT signals by introducing deep learning. Main highlights of this paper may include: i) An end-to-end DCS-based reconstruction framework is proposed, including angular sampling, multi-coset sampling, compressed sensing and learning sensing. By this way, high-fidelity and fast vibration reconstruction can be achieved, compared with classical CS algorithms; ii) A MCAS scheme is derived to adapt variable rotating speeds due to complex operation conditions; iii) A DCS model of BTT measurements is built in order domain, where a specific CNN model is built based on 
convolution layers with ReLU after BN; iv) The proposed method is better than classical reconstruction algorithms from the perspective of reconstruction accuracy and speed. However, this paper didn't discuss the generalization ability of the proposed DCS-based reconstruction method. Since the generalization ability of CNN models is not good, it deserves to explore other DNN models in future. In particular, transfer learning should be a promising solution [18, 32].

\section{Declarations}

\section{Availability of data and materials}

The datasets generated during and analysed during the current study are available from the corresponding author on reasonable request.

\section{Competing Interests}

The authors declare no competing financial interests.

\section{Funding}

This work was supported by the National Natural Science Foundation of China (Grant No. 51975206).

\section{Authors' contributions}

ZSC, HS and YPX designed the work; HS assisted with data sampling and experiment analysis; ZSC and CWL wrote the manuscript. All authors read and approved the final manuscript.

\section{Acknowledgements}

The authors would thank the experimental set-up provided by Beijing Key Laboratory of Health Monitoring and Self-recovery for High-end Mechanical Equipment, Beijing University of Chemical Technology.

\section{References}

[1] M Witoś. High sensitive methods for health monitoring of compressor blades and fatigue detection. The Scientific World Journal, 2013(29): 218460.

[2] J W Lin, J H Zhang, G C Zhang, et al. Aero-engine blade fatigue analysis based on nonlinear continuum damage model using neural networks. Chinese Journal of Mechanical Engineering, 2012, 25(2): 338-345.

[3] D Knappett, J Garcia. Blade tip timing and strain gauge correlation on compressor blades. Proceedings of the Institution of Mechanical Engineers Part G, Journal of Aerospace Engineering, 2008, 222(4): 497-506.

[4] J P Roberts. Comparison of tip timing with strain gauges for rotor blade vibration measurement. Proceedings of lecture series on tip timing and tip clearance problems in turbomachines. Von Karman Institute, Belgium 2007.

[5] S Heath, M Imregun. A survey of blade tip-timing measurement techniques for turbomachinery vibration. Journal of Engineering for Gas Turbines and Power, 1998, 120(4): 784-791.

[6] Z S Chen, H Sheng, Y M Xia, et al. A comprehensive review on blade tip timing-based health monitoring: status 
and future. Mechanical Systems and Signal Processing, 2021, 149: 107330.

[7] B Salhi, J Lardies, M Berthillier. Identification of modal parameters and aeroelastic coefficients in bladed disk assemblies. Mechanical Systems and Signal Processing, 2009, 23: 1894-1908.

[8] Z S Chen, Y M Yang, B Guo, et al. Blade damage prognosis based on kernel principal component analysis and grey model using subsampled tip-timing signals. Proceedings of the Institution of Mechanical Engineers, Part C: Journal of Mechanical Engineering Science, 2014, 228: 3178-3185.

[9] Z S Chen, Y M Yang, Y Xie, et al. Non-contact crack detection of high-speed blades based on principal component analysis and Euclidian angles using optical-fiber sensors. Sensors and Actuators A: Physical, 2013, 201: 66-72.

[10] Z S Chen, J He, C Zhan. Undersampled blade tip-timing vibration reconstruction under rotating speed fluctuation: uniform and nonuniform sensor configurations. Shock and Vibration, 2019, 2019: 8103216.

[11] Z S Chen, J H Liu, C Zhan, et al. Reconstructed order analysis-based vibration monitoring under variable rotation speed by using multiple blade tip-timing sensors. Sensors, 2018, 18: 3235.

[12] J Lin, Z Hu, Z S Chen, et al. Sparse reconstruction of blade tip-timing signals for multi-mode blade vibration monitoring. Mechanical Systems and Signal Processing, 2016, 85: 250-258.

[13] H L Xu, Z S Chen, Y M Yang, et al. Effects of crack on vibration characteristics of mistuned rotated blades. Shock and Vibration, 2017, 2017: 1785759.

[14] D Donoho. Compressed sensing. IEEE Transaction on Information Theory, 2006, 52(4):1289-306.

[15] H L Xu, Z S Chen, Y M Yang, et al. Multi-band blade vibration monitoring by blade tip-timing method based on compress sensing. Proceedings of the 1st World Congress on Condition Monitoring, 2017.

[16] Z S Chen, H Sheng, Y M Xia. Multi-coset angular sampling-based compressed sensing of blade tip-timing vibration signals under variable speeds. Chinese Journal of Aeronautics, 2021, 34(9): 83-93.

[17] M H Pan, F J Guan, H F Hu, et al. Compressed sensing based on dictionary learning for reconstructing blade tip timing signals. Proceedings of 2017 Prognostics and System Health Management Conference (PHM-Harbin), 9-12 July 2017.

[18] J Liang, L J Li, C K Zhao. A transfer learning approach for compressed sensing in 6G-IoT. IEEE Internet of Things Journal, 2021, 8(20): 15276-15283.

[19] R P Spada, R Nicoletti. Applying compressed sensing to blade tip timing data: A parametric analysis. Proceedings of the 10th International Conference on Rotor Dynamics, 2018, pp. 121-134.

[20] P Agrawal, D Chaudhary, V Madaan, et al. Automated bank cheque verification using image processing and deep learning methods. Multimedia Tools and Applications, 2021, 80: 5319-5350.

[21] V Hadi, H M Armita. Persian speech recognition using deep learning. International Journal of Speech Technology, 2020, 23(4):893-905 . 
[22] E Zisselman, A Adler, M Elad. Compressed learning for image classification: a deep neural network approach. Book Chapter: Handbook of Numerical Analysis, 2018, 19: pp. 3-17.

[23] J C Ye, Y S Han. Deep convolutional framelets: a general deep learning for inverse problems. SIAM Journal on Imaging Sciences, 2017, 11(2): 991-1048.

[24] Y K Gu, L Zeng, G Q Qiu. Bearing fault diagnosis with varying conditions using angular domain resampling technology, SDP and DCNN. Measurement, 2020, 156: 107616.

[25] E J Candès, J Romberg, T Tao. Robust uncertainty principles: exact signal reconstruction from highly incomplete frequency information. IEEE Transaction on Information Theory, 2006, 52: 489-509.

[26] J C Emmanuel. The restricted isometry property and its implications for compressed sensing. Comptes Rendus Mathematique, 2008, 346(9-10): 589-592

[27] S Ioffe S, C Szegedy. Batch normalization: accelerating deep network training by reducing internal covariate shift. Proceedings of International Conference on Machine Learning, 2015: pp. 448-456.

[28] G Chen, P Chen, Y Shi, et al. Rethinking the usage of batch normalization and dropout in the training of deep neural networks, arXiv:1905.05928, 2019.

[29] G E. Hinton, N Srivastava, A Krizhevsky, et al. Improving neural networks by preventing co-adaptation of feature detectors, arXiv:1207.0580, 2012.

[30] S Park, N Kwak. Analysis on the dropout effect in convolutional neural networks. Proceedings of Asian Conference on Computer Vision, Cham, 2016: pp. 189-204.

[31] C Y Zhou, H F Hu, F J Guan, et al. Modelling and simulation of blade tip timing uncertainty from rotational speed fluctuation. Proceedings of Prognostics and System Health Management Conference (PHM-Harbin), 9-12 July 2017, Harbin, China.

[32] D L Xu, L Du, H W Liu, et al. Compressive sensing of stepped-frequency radar based on transfer learning. IEEE Transactions on Signal Processing, 2015, 63(12): 3076-3087. 